\title{
Propuesta de indicadores para la vigilancia de la salud ocupacional en América Latina y el Caribe
}

\author{
Pamela Merino-Salazar, ${ }^{1}$ Cecilia Cornelio, ${ }^{2}$ Maria Lopez-Ruiz ${ }^{3}$ y Fernando G. \\ Benavides ${ }^{4}$ en nombre de la Red Experta en Encuestas de Condiciones de \\ Trabajo, Empleo y Salud (RED ECoTES)
}

Forma de citar Merino-Salazar P, Cornelio C, Lopez-Ruiz M, Benavides FG, Red Experta en Encuestas de Condiciones de Trabajo, Empleo y Salud. Propuesta de indicadores para la vigilancia de la salud ocupacional en América Latina y el Caribe. Rev Panam Salud Publica. 2018;42:e125. https://doi.org/10.26633/ RPSP.2018.125

RESUMEN

Este artículo propone un conjunto básico y común de indicadores para la vigilancia en salud ocupacional en América Latina y el Caribe e identifica las fuentes de datos disponibles para su medición. Para ello, se llevó a cabo un proceso de consenso de la Red Experta en Encuestas sobre Condiciones de Trabajo, Empleo y Salud (RED ECoTES) en dos etapas. En la primera etapa, se consensuó un primer listado de indicadores, y en la segunda etapa, se identificaron las fuentes de datos y sus características, con el fin de facilitar la comprobación de la factibilidad del cálculo real de cada indicador en 20 países de la Región. La propuesta del conjunto básico de indicadores, y sus definiciones, está conformado por 13 indicadores distribuidos en cuatro dimensiones: condiciones de empleo (tres indicadores), condiciones de trabajo (cuatro indicadores), recursos y actividades preventivas (dos indicadores) y salud (cuatro indicadores). Las principales fuentes disponibles para calcular estos indicadores en la Región fueron las encuestas permanentes de hogares, las encuestas de condiciones de trabajo, empleo y salud, las estadísticas vitales, y los sistemas de notificación de lesiones por accidentes de trabajo. En la Región existen datos para un número relevante de los indicadores propuestos. Sin embargo, un número importante de aquellos orientados a las condiciones de trabajo y actividades preventivas están ausentes en la mayoría de países. La vigilancia efectiva de la salud ocupacional en la Región exige impulsar decididamente las encuestas sobre condiciones de empleo, trabajo y salud en cada uno de los países que la conforman.

Palabras clave Indicadores de salud; vigilancia epidemiológica; sistema de información; salud de los trabajadores.

La definición y evaluación de políticas públicas para la prevención de los daños a la salud y la promoción del bienestar

\footnotetext{
Facultad de Ciencias del Trabajo y Comportamiento Humano, Universidad Internacional SEK, Quito, Ecuador. Enviar correspondencia a Pamela Merino-Salazar, dramerinos@gmail.com
}

de la población trabajadora en América Latina y el Caribe requiere del fortalecimiento de los sistemas de información

\footnotetext{
Superintendencia de Riesgos del Trabajo, Ministerio de Trabajo, Empleo y Seguridad Social, Buenos Aires, Argentina.

3 CIBER Epidemiología y Salud Pública (CIBERESP), Madrid, España.
}

en salud ocupacional. En este sentido, es necesario disponer de información de calidad, accesible, armonizada y puntual

4 Centro de Investigación en Salud Laboral UPF/ IMIM-PSMar, Barcelona, España.

Este es un artículo de acceso abierto distribuido bajo los términos de la licencia Creative Commons Attribution-NonCommercial-NoDerivs 3.0 IGO, que permite su uso, distribución y reproducción en cualquier medio, siempre que el trabajo original se cite de la manera adecuada. No se permiten modificaciones a los artículos ni su uso comercial. Al reproducir un artículo no debe haber ningún indicio de que la OPS o el artículo avalan a una organización o un producto específico. El uso del logo de la OPS no está permitido. Esta leyenda debe conservarse, junto con la URL original del artículo. 
que permita la comparabilidad entre territorios y en el tiempo, para su uso tanto en el ámbito nacional como regional. De hecho, la Organización Panamericana de la Salud (OPS) y la Organización Mundial de la Salud (OMS), a través del "Plan de acción sobre la salud de los trabajadores 2015-2025", reconocen dicha mejora como un objetivo prioritario a alcanzar a lo largo de este período (1). La información generada por los sistemas de información debe ser presentada como indicadores de fácil manejo e interpretación, sobre todo, para la gestión de políticas públicas $(2,3)$.

Disponer de información comparable entre países permite tanto establecer puntos de referencia para la evaluación de políticas públicas como identificar posibilidades de mejora. Algunas propuestas ya se han definido a nivel internacional, como por ejemplo la realizada en el año 2001 por un grupo del Instituto Finlandés en Salud Ocupacional (FIOH, por sus siglas en inglés), basada en indicadores para la vigilancia de la salud ocupacional en los países de la Unión Europea (4). Asimismo, la Organización Iberoamericana de Seguridad Social elaboró, en 2013, el Sistema Armonizado de Indicadores de Siniestralidad Laboral en Iberoamérica (SIARIN), si bien hasta la fecha no ha tenido la adhesión y el uso esperados (5).

Ahora bien, una vigilancia integral de la salud de la población trabajadora requiere disponer de indicadores que aborden las distintas dimensiones de la relación empleo y salud (6). Así, los datos para el cálculo de los mismos procederán de distintas fuentes. En este sentido, las encuestas de condiciones de trabajo, empleo y salud (ECTES) constituyen una de las principales e innovadoras fuentes de datos en la Región (7). Estas encuestas, a través de cuestionarios aplicados a muestras representativas, recogen la situación de las condiciones de trabajo y empleo, así como la percepción que la población trabajadora tiene sobre su estado de salud (8). Además, según el país, están disponibles otras fuentes de datos, como las encuestas de hogares $(9,10)$, que pueden aportar información sobre una o varias dimensiones de la salud ocupacional. Por otra parte, también existen los registros de los sistemas de seguridad social $\mathrm{y}$ de las administraciones ministeriales de trabajo y/o de salud, que recogen de manera sistemática información sobre lesiones por accidentes de trabajo y enfermedades profesionales.

Teniendo en cuenta estas premisas, los objetivos de este trabajo son, por un lado, proponer un conjunto básico y común de indicadores para la vigilancia en salud ocupacional en América Latina y el Caribe $\mathrm{y}$, por otro lado, identificar las fuentes de datos disponibles para su medición.

\section{MATERIALES Y MÉTODOS}

La propuesta del conjunto de indicadores es resultado de un proceso de consenso de 27 profesionales que conforman la Red Experta en Encuestas sobre Condiciones de Trabajo, Empleo y Salud (RED ECoTES). Dicha Red está compuesta por profesionales de la medicina, enfermería, odontología, psicología, sociología e ingeniería con formación en epidemiología, investigación en salud pública y ocupacional, y en las distintas ramas de la salud y seguridad, con responsabilidad en la toma de decisiones. Los profesionales proceden de universidades, centros de investigación y centros colaboradores de la OPS/OMS, así como de la administración pública de diversos países de América Latina y el Caribe (Argentina, Brasil, Chile, Colombia, Costa Rica, Ecuador, Honduras, Panamá, Perú, Puerto Rico y Uruguay), Estados Unidos y España, y fueron convocados por el alto nivel científico y técnico de la institución en el orden nacional y en el internacional; el lugar destacado que ocupa en la estructura sanitaria, de trabajo, científica y docente del país; y la alta calidad de su dirección científica y técnica, así como su capacidad multiplicadora del conocimiento, entre otros atributos. La Red se constituyó en 2014 en Buenos Aires con el objetivo de contribuir a mejorar los sistemas de información de salud ocupacional en Iberoamérica. Hasta la fecha, y después del encuentro inicial en Buenos Aires, se han celebrado tres encuentros: Belo Horizonte en 2015, Lima en 2016 y San José en 2017.

El proceso de consenso fue llevado a cabo en dos etapas (figura 1). El objetivo de la primera etapa, alcanzado durante

\section{FIGURA 1. Proceso de consenso del conjunto básico y común de indicadores para la vigilancia en salud ocupacional en América Latina y el Caribe}

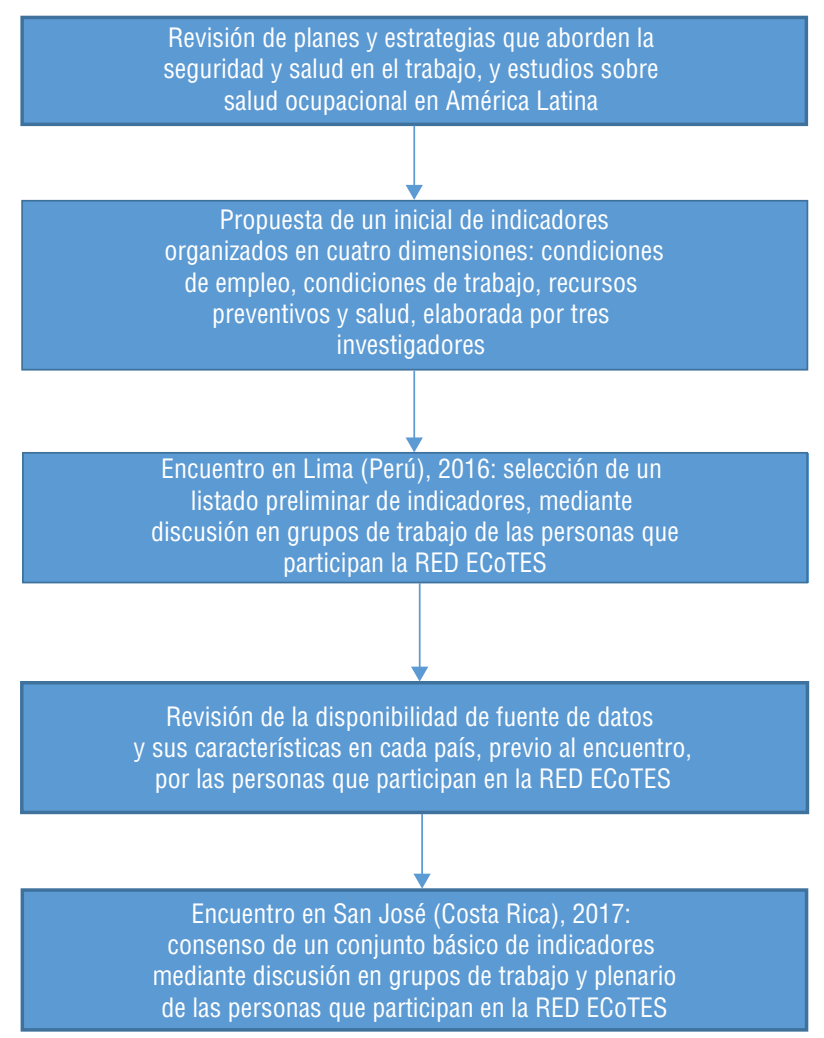

RED ECoTES, Red Experta en Encuestas de Condiciones de Trabajo, Empleo y Salud. Fuente: Elaboración propia. 
el encuentro en Lima, fue consensuar un listado preliminar de indicadores. De esta manera, previo al encuentro, tres investigadores de la Red establecieron un listado inicial de indicadores, cuya selección se basó en la metodología empleada por el FIOH, enfocada en una selección orientada por la evidencia científica (4). Así, partiendo de un modelo conceptual antes propuesto (6), se propusieron al inicio 22 indicadores ordenados en las cuatro dimensiones sugeridas en el modelo: 1) condiciones de empleo, que trata de captar el contexto socio-laboral, específicamente la situación del mercado de trabajo y las relaciones laborales, 2) condiciones de trabajo, relacionadas con el medio ambiente físico y organizativo de los lugares de trabajo, 3) recursos y actividades preventivas, que informa sobre la organización de los recursos y actividades en el centro de trabajo relacionadas con la prevención de los riesgos laborales y la participación de la población trabajadora y 4) salud, referida al estado general de salud y los daños en términos de lesiones y mortalidad. De la misma manera, en este proceso inicial de selección se examinaron planes y estrategias relacionadas con la salud y seguridad en el trabajo. En primer lugar, se consideró el objetivo 8 de la Agenda 2030 para el Desarrollo Sostenible de las Naciones Unidas que tiene como finalidad "promover el crecimiento económico sostenido, inclusivo y sostenible, el empleo pleno y productivo y el trabajo decente para todos" (11). Así, los indicadores de condiciones de empleo se seleccionaron con base en las metas planteadas en este objetivo. También se consideró el "Plan de Acción sobre Salud de los Trabajadores 2015-2025", cuya meta es fortalecer la respuesta del sector salud y de los actores involucrados para brindar atención integral a la salud de la población trabajadora, y disminuir las desigualdades en salud (1). Por otra parte, la selección de los indicadores de condiciones de trabajo (riesgos de seguridad, higiene, ergonomía y psicosociales) se basó en un estudio que midió la frecuencia de exposición a las distintas condiciones de trabajo desfavorables en muestras amplias de la población trabajadora en países de América Latina, para determinar los indicadores más relevantes a ser incorporados (12). Por último, se tuvo en cuenta el Cuestionario básico para las Encuestas sobre Condiciones de Trabajo, Empleo y
Salud en América Latina y el Caribe (CTESLAC) (7), del que se seleccionaron preguntas de condiciones de trabajo que podrían ser indicadores comparables y "trazadores" de estas condiciones en la Región.

Este primer listado se envió a la RED, previo al encuentro en Lima, por correo electrónico junto a los documentos y bibliografía que guiaron esta selección. Asimismo, se les solicitó revisar las estrategias nacionales de seguridad y salud en el trabajo. Con estos antecedentes, durante el encuentro se estructuraron cuatro grupos de trabajo según las dimensiones propuestas, y se llegó a un consenso inicial de 19 indicadores.

En una segunda etapa, previo al último encuentro celebrado en San José de Costa Rica, se revisó la disponibilidad de fuente de datos y sus características, con el fin de comprobar la factibilidad del cálculo de cada indicador, en 20 países de la Región: Argentina, Bolivia, Brasil, Chile, Colombia, Costa Rica, Cuba, Ecuador, El Salvador, Guatemala, Honduras, México, Nicaragua, Panamá, Paraguay, Perú, Puerto Rico, República Dominicana, Uruguay y Venezuela. En dicha revisión participaron todas las personas de la Red aportando los conocimientos sobre sus respectivos países y, además, se buscó la información necesaria en las administraciones de trabajo y salud e institutos nacionales de estadísticas, así como en organismos internacionales. Durante el encuentro, por medio de grupos de trabajo y en plenario, se consensuó un conjunto básico de indicadores para la Región y se identificaron las fuentes de datos de las que en principio se podrán obtener.

\section{RESULTADOS}

En el cuadro 1 se muestra el conjunto básico de 13 indicadores propuesto, distribuido en cuatro dimensiones: condiciones de empleo (tres indicadores), condiciones de trabajo (cuatro indicadores), recursos y actividades preventivas (dos indicadores) y salud (cuatro indicadores).

Esta propuesta incluye, además, la recomendación de desagregar dichos indicadores, en la medida que los datos estén disponibles, por sexo, grupos de edad, actividad económica, ocupación, y unidades geográficas más pequeñas en cada país (ej., departamentos, provincias o estados).

\section{Fuentes de datos sobre indicadores de condiciones de empleo}

Las fuentes de datos para los indicadores de condiciones de empleo se identificaron para los 20 países estudiados: Argentina (13-15), Bolivia (16, 17), Brasil $(18,19)$, Chile (20-22), Colombia (23), Costa Rica (24, 25), Cuba (26), Ecuador (27), El Salvador $(25,28)$, Guatemala $(25,29)$, Honduras $(25,30)$, México (31), Nicaragua $(25,32)$, Panamá (25, 33), Paraguay (34), Perú (35), Puerto Rico (36), República Dominicana (37), Uruguay (38) y Venezuela (39) En casi todos ellos, las principales fuentes fueron las encuestas de hogares -del tipo de propósitos múltiples- y las encuestas de empleo, que son usualmente gestionadas por los institutos nacionales de estadística. En general, los resultados de estas encuestas fueron accesibles y publicados de forma periódica. La mayoría de las instituciones gestoras, a través de sus páginas electrónicas, publica la información de dichas encuestas en cuadros estadísticos con diferentes niveles de desagregación o como información de tipo documental. Además, se identificó la posibilidad de obtener los datos desagregados mediante la descarga directa de las bases de datos de las encuestas en la mitad de los países estudiados.

Los datos sobre condiciones de empleo también son difundidos por la Comisión Económica para América Latina y el Caribe (CEPAL) y la Organización Internacional del Trabajo (OIT), por medio de CEPALSTAT (40) e ILOSTAT (41), que son las bases de datos e indicadores que consolidan los datos procedentes de las fuentes oficiales de los países.

\section{Fuentes de datos sobre indicadores de condiciones de trabajo, y recursos $\mathrm{y}$ actividades preventivas}

Para estas dimensiones, las principales fuentes de datos identificadas fueron las ECTES de la Región que se han realizado en 11 países: Colombia $(42,43)$, Argentina (15), Chile (21), Panamá, Costa Rica, Nicaragua, Honduras, El Salvador, Guatemala (25), Uruguay (44) y Perú. Las ECTES han sido gestionadas, en su mayoría, por las administraciones nacionales de trabajo o de salud y, en algunos casos, por organismos internacionales y universidades. Algunos resultados de estas encuestas están disponibles en los informes institucionales correspondientes. 
CUADRO 1. Conjunto básico de indicadores (y sus definiciones) para la vigilancia de la salud ocupacional en América Latina y el Caribe

\begin{tabular}{|c|c|}
\hline Indicador & Definición \\
\hline \multicolumn{2}{|l|}{ Condiciones de empleo } \\
\hline Tasa de ocupación & Relación entre la cantidad de trabajadores/as ocupados y el total de personas en edad de trabajar \\
\hline $\begin{array}{l}\text { Porcentaje de personas trabajadoras afiliadas } \\
0 \text { cubiertas por la seguridad social }\end{array}$ & $\begin{array}{l}\text { Cantidad relativa de trabajadores/as que tiene descuento, aporta, o están afiliados/as o registrados en algún } \\
\text { sistema de pensión por jubilación u otra contingencia en la seguridad social o caja de seguros respecto al } \\
\text { total de ocupados. }\end{array}$ \\
\hline Tasa de desempleo & $\begin{array}{l}\text { Relación entre la población desocupada y la población económicamente activa (conjunto de personas que } \\
\text { trabajan o buscan trabajo) }\end{array}$ \\
\hline \multicolumn{2}{|l|}{ Condiciones de trabajo } \\
\hline $\begin{array}{l}\text { Porcentaje de personas trabajadoras expuestas a } \\
\text { ruido elevado }\end{array}$ & $\begin{array}{l}\text { Cantidad relativa de trabajadores/as encuestados que respondieron "siempre" o "muchas veces" a la } \\
\text { pregunta: en su trabajo principal y en una jornada habitual ¿Está expuesto a un nivel de ruido que le obliga } \\
\text { a elevar la voz para conversar con otra persona?a }\end{array}$ \\
\hline $\begin{array}{l}\text { Porcentaje de personas trabajadoras en contacto con } \\
\text { sustancias químicas nocivas/tóxicas }\end{array}$ & $\begin{array}{l}\text { Cantidad relativa de trabajadores/as encuestados que respondieron "siempre" o "muchas veces" a la } \\
\text { pregunta: en su trabajo principal y en una jornada habitual ¿Manipula, aplica o está en contacto con } \\
\text { sustancias químicas nocivas/tóxicas?a }\end{array}$ \\
\hline $\begin{array}{l}\text { Porcentaje de personas trabajadoras que realizan } \\
\text { movimientos repetitivos }\end{array}$ & $\begin{array}{l}\text { Cantidad relativa de trabajadores/as encuestados que respondieron "siempre" o "muchas veces" a la } \\
\text { pregunta: en su trabajo principal y en una jornada de trabajo habitual ¿Con qué frecuencia realiza } \\
\text { movimientos repetitivos, casi idénticos con los dedos, manos o brazos cada pocos segundos?a }\end{array}$ \\
\hline $\begin{array}{l}\text { Porcentaje de personas trabajadoras que tienen que } \\
\text { trabajar muy rápido }\end{array}$ & $\begin{array}{l}\text { Cantidad relativa de trabajadores/as encuestados que respondieron "siempre" o "muchas veces" a la } \\
\text { pregunta: en su trabajo principal y en una jornada habitual ¿Con qué frecuencia tiene que trabajar } \\
\text { muy rápido?a }\end{array}$ \\
\hline \multicolumn{2}{|l|}{ Recursos y actividades preventivas } \\
\hline $\begin{array}{l}\text { Porcentaje de personas trabajadoras que tienen acceso } \\
\text { a un servicio de prevención de riesgos laborales o salud } \\
\text { ocupacional }\end{array}$ & $\begin{array}{l}\text { Cantidad relativa de trabajadores/as encuestados que respondieron "sí" a la pregunta "En su centro } \\
\text { de trabajo, ¿Tiene acceso a un servicio de prevención de riesgos laborales o de salud laboral?a }\end{array}$ \\
\hline $\begin{array}{l}\text { Porcentaje de personas trabajadoras que en su centro de } \\
\text { trabajo cuentan con representación gremial en materia de } \\
\text { salud y seguridad en el trabajo }\end{array}$ & $\begin{array}{l}\text { Cantidad relativa de trabajadores/as encuestados que respondieron "sí" a la pregunta En su centro de } \\
\text { trabajo, ¿existe un delegado de prevención, comisión o comité de salud y seguridad o higiene en el } \\
\text { trabajo?a }\end{array}$ \\
\hline \multicolumn{2}{|l|}{ Salud } \\
\hline $\begin{array}{l}\text { Tasa de incidencia de lesiones no mortales por accidentes } \\
\text { de trabajo por } 1000 \text { personas trabajadoras }\end{array}$ & $\begin{array}{l}\text { Número total de lesiones no mortales por accidente de trabajo notificadas a la autoridad laboral en el } \\
\text { periodo de un año, entre el total de la población expuesta o cubierta por el seguro social, expresada por } \\
1000 \text { trabajadores/as. }\end{array}$ \\
\hline $\begin{array}{l}\text { Tasa de incidencia de lesiones mortales por accidentes de } \\
\text { trabajo por } 100000 \text { personas trabajadoras }\end{array}$ & $\begin{array}{l}\text { Número total de accidentes de trabajo mortales notificados a la autoridad laboral en el periodo de un } \\
\text { año, entre el total de la población expuesta o cubierta por el seguro social, expresada por } 100000 \\
\text { trabajadores/as. }\end{array}$ \\
\hline $\begin{array}{l}\text { Porcentaje de personas trabajadoras con mala salud } \\
\text { autopercibida }\end{array}$ & $\begin{array}{l}\text { Cantidad relativa de trabajadores encuestados que respondieron "regular", "mala" o "muy mala" a la } \\
\text { pregunta ¿Cómo considera usted que es su estado de salud en general? }\end{array}$ \\
\hline $\begin{array}{l}\text { Tasa de mortalidad general para el grupo etario de } 15 \text { a } \\
65 \text { años }\end{array}$ & $\begin{array}{l}\text { Número total de defunciones por todas las causas, estimadas en la población de } 15 \text { a } 65 \text { años, entre el total } \\
\text { de esa población, expresada por } 100000 \text { habitantes, para el periodo seleccionado }\end{array}$ \\
\hline
\end{tabular}

Fuente: Elaboración propia a partir de los resultados del estudio

a Definiciones tomadas del Cuestionario para las Encuestas de Condiciones de Trabajo, Empleo y Salud (CTESLAC), válido también para otros cuestionarios que emplean una pregunta similar.

Sin embargo, hasta donde hemos podido comprobar, solo la base de datos de la encuesta de Centroamérica está disponible bajo solicitud. Para otros países, como Brasil, están disponibles datos sobre exposiciones a riesgos laborales como ruido o productos tóxicos en la Pesquisa Nacional de Saúde (45).

\section{Fuentes de datos sobre indicadores de salud}

Por último, se proponen las fuentes de datos para los indicadores de salud. La existencia de datos para calcular las tasas de incidencia de lesiones mortales y no mortales por accidentes de trabajo es elevada y, en su mayoría, proceden de las instituciones de la seguridad social: Argentina (46), Brasil (47), Chile (48), Colombia (49), Costa Rica (50), Ecuador (51), El Salvador (52), Honduras (53), México (54), Nicaragua (55), Perú (56), República Dominicana (57), Uruguay (58) y Venezuela (59). Sin embargo, algunas fuentes solo consideran a la población trabajadora con empleos formales o afiliada a la seguridad social. Además, en algunos países, la información se presenta poco desagregada por variables sociodemográficas o laborales en la página electrónica de las instituciones correspondientes, como en Colombia, Ecuador, El Salvador y Honduras, e incluso en algunos casos no se ha logrado acceder a las fuentes de datos respectivas (Bolivia, Guatemala, Paraguay, Panamá y Cuba).

Los datos para calcular el indicador de salud autopercibida está disponible en la mayoría de los países que cuentan con ECTES $(21,25,43)$, y también en algunos de los que han realizado encuestas de salud (60-64). Por otro lado, en la mayoría de países estudiados, las fuentes de datos para calcular la tasa de mortalidad general son las estadísticas vitales, difundidas por las administraciones de salud o los institutos nacionales de estadística.

De igual manera, ILOSTAT ofrece las tasas de incidencia de lesiones mortales y no mortales por accidentes de trabajo 
en la mayoría de países de la Región. Por su parte, la OPS consolida los datos sobre mortalidad proporcionada por los diferentes países y los difunde a través del informe anual "Situación de Salı̀ de las Américas: Indicadores Básicos" mediante el Sistema de Información de Mortalidad Regional (65).

La lista detallada de todas las fuentes empleadas y sus características se puede obtener previa solicitud a la autora de correspondencia de este artículo.

\section{DISCUSIÓN}

Los resultados de este trabajo de carácter documental suponen un primer paso significativo para establecer un sistema de vigilancia de la salud de las personas trabajadoras en América Latina y el Caribe. La propuesta incluye un conjunto básico de 13 indicadores que integra las diferentes dimensiones de la salud ocupacional. A su vez, contribuye con la identificación de numerosas fuentes de datos que pueden ser potencialmente empleadas para la medición de estos indicadores. Sin embargo, las fuentes existentes se caracterizan por estar fragmentadas en diversas instituciones gestoras y por la dificultad en el acceso a sus datos en numerosas ocasiones.

El conjunto de indicadores consensuado podría, por un lado, proporcionar información esencial para la vigilancia de las condiciones de trabajo y de empleo, recursos y actividades preventivas y el estado de salud de la población trabajadora dentro de cada país $y$, por otro lado, permitir las comparaciones entre países y con otras regiones del mundo. En esta propuesta, se ha incluido un conjunto básico de indicadores para los cuales hay datos disponibles en la mayor parte de países como, por ejemplo, tasa de ocupación, tasa de desempleo, tasa de incidencia de lesiones mortales y no mortales por accidentes de trabajo y tasa de mortalidad general para el grupo etario de 15 a 65 años. Asimismo, con el propósito de incluir información relevante para la toma de decisiones en salud ocupacional, se han incorporado indicadores sobre condiciones de trabajo y actividades preventivas, a pesar de no disponer de datos para su medición en gran parte de los países. Estos datos podrían estar disponibles en un futuro a través de la implementación de las ECTES en los países de la Región que aún no las tienen o mediante la incorporación de módulos específicos en otro tipo de encuestas nacionales, ya sea encuestas de propósitos de múltiples, de empleo o de salud, entre otras.

Al emplear las fuentes descritas para la medición de los indicadores cabe tener en cuenta, además, su periodicidad. Por una parte, las fuentes de datos sobre empleo, en general, son encuestas continuas y publican la información con una periodicidad corta. De forma similar, las fuentes sobre lesiones por accidentes de trabajo proporcionan información en base a un calendario fijo anual. Por otra parte, a pesar de ser una herramienta prometedora para la vigilancia integral de la salud ocupacional, en la actualidad no se han llevado a cabo las ECTES en la mayoría de países de la Región y, en los países en los que se logrado realizar al menos una primera edición, aún no se cuenta con el apoyo necesario para establecer una periodicidad de ejecución. Esto constituye un limitante significativo para la toma de decisiones en base a información puntual y actualizada. Cabe señalar que, si se desea evaluar las políticas de seguridad y salud en el trabajo, es necesario disponer de las tendencias temporales de los indicadores.

La exhaustiva revisión documental pone en evidencia dos hechos notables para la vigilancia de la salud ocupacional, como son, en primer lugar, que las principales fuentes de datos dependen de administraciones laborales o económicas (institutos de estadísticas), y no tanto de la administración de salud. En segundo lugar, los resultados muestran que hay un amplio espacio para mejorar la información disponible, sobre todo en relación a las condiciones de trabajo y recursos preventivos. En este sentido, la realización de ECTES periódicas, como ocurre en la Unión Europea cada cinco años (66), en los países de la Región que cuentan con una única edición o en los que no se ha realizado ninguna todavía, como Bolivia, Brasil, México o Venezuela, entre otros, es urgente, pues es la única fuente que nos permite conocer y monitorear las condiciones de trabajo, la salud y los recursos preventivos en los centros de trabajo. Así, existe la necesidad de impulsar decididamente la realización de ECTES en cada uno de los países de la Región, basadas en cuestionarios y metodologías que las hagan comparables. A su vez, la incorporación de las ECTES permitiría alcanzar poblaciones laborales a las que no se tiene acceso de otro modo, como es la población con empleo informal, que en América Latina es un grupo en particular vulnerable y muy amplio dentro de la población trabajadora (67). Asimismo, este esfuerzo compromete a la RED ECoTES. Esta se ha expresado en intensificar las medidas para poner a disposición de los estados y demás actores sociales los instrumentos consensuados para la evaluación de las condiciones de trabajo y su impacto en la salud de la población trabajadora, mediante la promoción de una agenda de Encuestas en los países que permita el monitoreo de las políticas respectivas.

Ahora bien, esta propuesta es un primer paso necesario, pero no suficiente, pues ahora queda comprobar la comparabilidad de los datos disponibles en cada país. En este sentido, confiamos que la mayoría de los países, si no todos, hayan incorporado las definiciones establecidas por las organizaciones internacionales, como la OIT, para la recogida y tabulación de datos básicos como ocupación, desempleo o accidentes de trabajo, entre otros. Otras resultarán más complicadas, como puede ser la referida al seguro social, donde la diversidad de definiciones puede dificultar su comparabilidad. Asimismo, respecto a las condiciones de trabajo y las actividades preventivas, al provenir de las ECTES, su comparabilidad es en la actualidad limitada, como ya se ha comprobado (12). Sin embargo, recientemente se ha desarrollado una propuesta de un cuestionario básico y unos criterios metodológicos para incrementar la comparabilidad de futuras encuestas en la Región (7).

Entre las limitaciones del presente trabajo hay que señalar que no se incluyen todos los países de la Región, pues para hacer factible este primer trabajo de documentación nos hemos centrado en aquellos que tenían el español o el portugués como idiomas oficiales. Por otro lado, la dificultad en el acceso a los datos ha limitado de manera significativa nuestro trabajo. En este sentido, en la mayoría de los países se pueden identificar las fuentes de datos, pero se accede a la información a través de memorias, boletines, notas de prensa o informes, entre otras, publicados en las páginas web de las instituciones.

Una de las mayores fortalezas de este trabajo es la participación de la Red ECoTES, conformada por un grupo multidisciplinar de especialistas en salud 
ocupacional de la mayoría de los países de la Región, pues el proceso de consenso y la búsqueda de fuentes de datos han estado orientados por personas con experiencia en su propio país.

En conclusión, la propuesta de los indicadores consensuados para la vigilancia de la salud de la población trabajadora podría ser útil para el diseño y evaluación de las políticas públicas en Seguridad y Salud en el Trabajo de la Región. Sin embargo, hoy en día, la medición de estos indicadores depende de fuentes de datos no siempre accesibles, a veces dispersas en diversas instituciones, en ocasiones, aún no consolidadas y que, con frecuencia, excluyen a la población con empleo informal. Por lo tanto, mejorar estas fuentes, sobre todo las ECTES, constituye un reto imprescindible si queremos disponer de sistemas nacionales y regionales de vigilancia de la salud de la población trabajadora, necesarios para valorar los efectos de la globalización y las normativas nacionales e internacionales, así como las políticas públicas en la salud de las personas que trabajan de manera remunerada.

Agradecimientos Este proyecto ha sido posible gracias a la contribución de diversas instituciones con la organización, los recursos y el interés por sostener las actividades de Red ECoTES, destacando entre ellas al Centro de investigación en Salud Laboral, Universitat Pompeu Fabra de Barcelona- España, a la OPS, a los Centros Colaboradores en Salud Ocupacional de la OPS del Instituto Regional de Estudios en Sustancias Tóxicas de la Universidad Nacional de Costa Rica (IRET-UNA) y de la Escuela de Salud Pública de Houston de la Universidad de Texas, a la Superintendencia de Riesgos del Trabajo, Ministerio del Trabajo, Empleo y Seguridad Social de Argentina, a la Universidad Federal de Minas Gerais de Brasil, al Centro Nacional de Salud Ocupacional y Protección del Ambiente para la Salud, a la Sociedad Peruana de Salud Ocupacional y a la Universidad

\section{REFERENCIAS}

1. Organización Panamericana de la Salud (OPS). Plan de Acción sobre la Salud de los Trabajadores 20152025. 2017. Disponible en: http://iris. paho.org/xmlui / bitstream/handle / 123456789/33983/CD54_10Rev.\%201-spa. pdf?sequence $=1 \&$ is Allowed $=y$ Acceso el 20 de marzo de 2017.

2. Benavides FG, Pérez G, Gispert, R. Revisión de las fuentes de datos disponibles y propuesta de indicadores de vigilancia en salud laboral. Arch Prev Riesgos Labor. 2002;5(02):62-7.

3. Health Metrics Network, World Health Organization (WHO). Framework and standards for country health information systems. Geneva: WHO; 2008. Disponible en: http: / / www.who.int/healthinfo / country_monitoring_evaluation/whohmn-framew ork-standards-chi.pdf Acceso el 20 de marzo de 2017.

4. Rantanen J, Kauppinen T, Toikkanen J, Kurppa K, Lehtinen S, Leino T. Work and health country profiles. Country profiles and national surveillance indicators in occupational health and safety. Helsinki: Finish Institute of Occupational Health; 2001. Disponible en: http://www.who. int/occupational_health/publications / eurprofiles/en/ Acceso el 10 de febrero de 2017.

5. Organización Iberoamericana de Seguridad Social (OISS). Documento técnico para la implantación del Sistema Armonizado de Indicadores Básicos de Siniestralidad y Salud Laboral en Iberoamérica. OISS; 2013. Disponible en: http: / / www.oiss.org/estrategia / IMG/pdf/SIARIN.pdf Acceso el 10 de febrero de 2017.

6. Benavides FG, García AM, Ruiz FrutosCarlos. Capítulo 1. La salud y sus determinantes. En: Salud Laboral: conceptos y técnicas para la prevención de riesgos laborales. Barcelona: Elsevier, Masson; 2014:3-15.

7. Benavides FG, Merino-Salazar P, Cornelio C, Lima E, Avila A. Red de Expertos en Encuestas sobre Condiciones de Trabajo, Empleo y Salud. Cuestionario y criterios metodológicos para Encuestas sobre Condiciones de Trabajo, Empleo y Salud en América Latina y el Caribe. Cad Saude Publica. 2016;323(9):e00210715.

8. Iñiguez M, Agudelo-Suarez A, CamposSerna J, Cornelio C, Benavides F. Encuestas de condiciones de trabajo y salud: su utilización en la investigación en salud laboral. Med Segur Trab. 2012;58(228):205-15.

9. Comisión Económica para América Latina y el Caribe (CEPAL). Hacia un sistema integrado de encuestas de hogares en los países de América Latina. Santiago de Chile: Naciones Unidas; 2001. Disponible en: http:/ / repositorio.cepal.org/bitstream / handle/11362/4707/1/S01010053_es.pdf Acceso el 5 de febrero de 2017.

10. Oficina Internacional del Trabajo (OIT), Departamento de Estadística. Estadística del trabajo y de la fuerza de trabajo: Informe para el debate en la Reunión de expertos en estadísticas del trabajo para el avance de las estadísticas sobre el empleo y el desempleo. Ginebra: OIT, 2013.
Peruana Cayetano Heredia. Agradecemos de manera especial al resto de personas expertas de la RED ECoTES que han contribuido al trabajo que se presenta en este artículo: Andrés A. Agudelo-Suarez, Amanda Alvarado Martínez, Marcelo Amable, Lucía Artazcoz, John Astete, Ada Ávila Assunção, Douglas Barraza, Dinora Bernal, Lino Carmenate Millán, George Delclòs, Lorena Funcasta, David Gimeno Ruiz de Porras, María José Itatí Iñiguez, Eduardo de Paula Lima, David Martínez-Iñigo, Adriane Mesquita de Medeiros, Lida Orta, Javier Pinilla, Marianela Rojas, Iselle Sabastizagal, Clelia Vallebuona, Gloria H. Villalobos y Alejandra Vives.

Conflicto de intereses Ninguno declarado por los autores.

Declaración Las opiniones expresadas en este manuscrito son responsabilidad del autor y no reflejan necesariamente los criterios ni la política de la RPSP/ PAJPH y/o de la OPS.
Disponible en: http://www.ilo.org/wcmsp5/groups/public/---dgreports / --stat/documents / meetingdocument/ wcms_202307.pdf Acceso el 15 de junio de 2017.

11. United Nations (UN). Transforming our world: the 2030 Agenda for Sustainable Development (A/RES/70/1). UN, 2015. Disponible en: https:// sustainabledevelopment.un.org/content/ documents / $21252030 \% 20$ Agenda $\% 20$ for $\% 20$ Sustainable $\% 20$ Development $\% 20$ web.pdf Acceso el 15 de junio de 2017.

12. Merino-Salazar P, Artazcoz L, Cornelio C, et al. Work and health in Latin America: results from the working conditions surveys of Colombia, Argentina, Chile, Central America and Uruguay. Occup Environ Med. 2017;74:432- 9.

13. Instituto Nacional de Estadística y Censos. República Argentina. Base de datos [Internet]. Buenos Aires: INDEC, 2018. Disponible en: https://www.indec.gob. ar/bases-de-datos.asp Acceso el 3 de junio de 2018.

14. Ministerio de Trabajo, Empleo y Seguridad Social de la República Argentina. Estudios, informes y estadísticas de Seguridad Social [Internet]. Disponible en: https: / / www.argentina.gob.ar/trabajo/ seguridadsocial/estudios Acceso el 4 de junio de 2018.

15. Superintendencia de Riesgos del Trabajo (SRT), Ministerio de Trabajo, Empleo y Seguridad Social de la República Argentina. Encuesta nacional a trabajadores, empleo, trabajo, condiciones $y$ 
medio ambiente laboral. Buenos Aires: SRT; 2013. Disponible en: http:// biblioteca.srt.gob.ar/Publicaciones/2013/ EncuestaNac2009.pdf. Acceso el 8 de diciembre de 2017

16. Instituto Nacional de Estadística del Estado Plurinacional de Bolivia. Encuesta de Empleo. Disponible en: https://www. ine.gob.bo/index.php/mercado-laboral/ introduccion-3 Acceso el 4 de junio de 2018.

17. Instituto Nacional de Estadística del Estado Plurinacional de Bolivia. Bases de datos de encuestas sociales. Disponible en: https://www.ine.gob.bo/index.php/ banco/base-de-datos-sociales Acceso el 3 de junio de 2018

18. Instituto Brasileiro de Geografia e Estatística. Pesquisa Mensal de Emprego. Disponible en: https://ww2.ibge.gov. br/home/estatistica/indicadores / trabalhoerendimento/pme_nova/default. shtm Acceso el 3 de junio de 2018.

19. Instituto Brasileiro de Geografia e Estatística. Pesquisa Nacional por Amostra de Domicílios. Disponible en: https://ww2. ibge.gov.br/home/estatistica/populacao/ trabalhoerendimento/pnad2015/default. shtm Acceso el 2 de junio de 2018.

20. Instituto Nacional de Estadísticas de Chile. Encuesta Nacional de Empleo [Internet]. Disponible en: http://www. ine.cl/estadisticas/laborales/ene Acceso el 4 de junio de 2018.

21. Ministerio de Salud de Chile. Primera encuesta nacional de empleo, trabajo, salud y calidad de vida de los trabajadores y las trabajadoras en Chile (ENETS 20092010) [Internet]. Santiago: Departamento de Epidemiología, Ministerio de Salud; 2011. Disponible en: http:/ / epi.minsal.cl/ encuesta-enets / Acceso el 7 de mayo 2018.

22. Ministerio de Desarrollo Social de Chile. Observatorio Social. Archivo histórico de Encuesta CASEN. Disponible en: http:// observatorio.ministeriodesarrollosocial. gob.cl/casen/casen_obj.php\# Acceso el 1 de junio de 2018

23. Departamento Administrativo Nacional de Estadística de Colombia. Visor de Microdatos. Archivo Nacional de Datos (ANDA) [Internet]. Disponible en: https: / / sitios.dane.gov.co/visor-anda / Acceso el 2 de junio de 2018.

24. Instituto Nacional de Estadística y Censos de Costa Rica. Encuesta Continua de Empleo [Internet]. Disponible en: http:// www.inec.go.cr/encuestas/encuestacontinua-de-empleo Acceso el 4 de junio de 2018.

25. Benavides FG, Wesseling C, Delclos GL, Felknor S, Pinilla J, Rodrigo F. Working conditions and health in Central America: a survey of 12,024 workers in six countries. Occup Environ Med. 2014;71:459-65.

26. Oficina Nacional de Estadística e Información de la República de Cuba. Sistema de Información Estadística Nacional. Disponible en: http://www. one.cu/sien2018.htm Acceso el 4 de junio de 2018.

27. Instituto Nacional de Estadística y Censos de Ecuador. Encuesta Nacional de Empleo, Desempleo y Subempleo. Disponible en: http: / /www.ecuadorencifras.gob.ec/ empleo-marzo-2018/ Acceso el 3 de junio de 2018.

28. Dirección General de Estadística y Censos de El Salvador. Publicaciones. Disponible en: http://www.digestyc.gob.sv/index. $\mathrm{php} /$ temas/des/ehpm/publicacionesehpm.html Acceso el 2 de junio de 2018.

29. Instituto Nacional de Estadística de Guatemala. Encuesta Nacional de Empleo e Ingresos. Disponible en: https://www. ine.gob.gt/index.php/encuestas/empleoe-ingresos Acceso el 4 de junio de 2018.

30. Instituto Nacional de Estadísticas de Honduras. Encuesta Permanente de Hogares. Disponible en: www.ine.gob. hn/index.php/component/content/ article?id=170 Acceso el 30 de abril de 2018.

31. Instituto Nacional de Geografía y Estadística de México. Encuesta Nacional de Ocupación y Empleo (ENOE). Disponible en: www.beta.inegi.org.mx/ proyectos/enchogares/regulares/enoe/. INEGI Acceso el 30 de abril de 2018.

32. Instituto Nacional de Información de Desarrollo de Nicaragua (INIDE). Informe de Empleo. Encuesta Continua de Hogares. IV Trimestre 2017. Managua: INIDE; 2018. Disponible en: www.inide. gob.ni/ Acceso el 30 de junio de 2018.

33. Instituto Nacional de Estadística y Censo de Panamá. Encuesta de Propósitos Múltiples. Instituto Nacional de Estadísticas y Censos. 2010. Disponible en: www. contraloria.gob.pa/inec Acceso el 4 de junio de 2018.

34. Dirección General de Estadística, Encuestas y Censos de Paraguay. Microdatos. Disponible en: http://www. dgeec.gov.py/microdatos/ Acceso el 4 de junio de 2018.

35. Instituto Nacional de Estadística e Informática de Perú. Microdatos. Disponible en: http://iinei.inei.gob.pe/ microdatos/index.htm Acceso el 4 de junio de 2018.

36. Departamento del Trabajo y Recursos Humanos de Puerto Rico. Información del mercado laboral de Puerto Rico [Internet]. Departamento de Trabajo y Recursos Humanos. Disponible en: http:// www.mercadolaboral.pr.gov / Tablas Estadisticas.aspx Acceso el 4 de junio de 2018.

37. Banco Central de la República Dominicana. Encuesta Nacional Continua de Fuerza de Trabajo. Disponible en: https:// www.bancentral.gov.do/estadisticas economicas / mercado_trabajo/encft / Acceso el 4 de junio de 2018.

38. Instituto Nacional de Estadística de Uruguay. Encuesta Continua de Hogares. Disponible en: http://www.ine.gub. uy/web/guest/encuesta-continua-dehogares1 Acceso el 4 de junio de 2018.

39. Ministerio del Poder Popular de Planificación de la República Bolivariana de Venezuela. Instituto Nacional de Estadística. Ficha Técnica de Encuesta de Hogares por Muestreo. Disponible en: http://www.ine.gov.ve/index.php? option $=$ com_content \&id $=333 \&$ Itemid=103 Acceso el 4 de junio de 2018.
40. Comisión Económica para América Latina y el Caribe. CEPALSTAT: Base de Datos y Publicaciones Estadísticas. Disponible en: http:/ / estadisticas.cepal.org/cepalstat/ Portada.html Acceso el 7 de mayo de 2018.

41. Organización Internacional del Trabajo. ILOSTAT [Internet]. Disponible en: http:/ / www.ilo.org/ilostat/faces/ilostat-home/ home?_adf.ctrl-state=z0ab8thh5_333\&_ afrLoop=7629113315209\#! Acceso el 7 de mayo de 2018.

42. Ministerio de Protección Social de Colombia. Primera Encuesta nacional de condiciones de salud y trabajo en el Sistema General de Riesgos Profesionales (I ENCST). Bogotá: Ministerio de Protección Social; 2007. Disponible en: http://www.oiss.org/estrategia/IMG/ pdf/I_encuesta_nacional_colombia2.pdf Acceso el 5 de noviembre de 2017.

43. Ministerio de Trabajo de Colombia. II Encuesta nacional de condiciones de seguridad y salud en el trabajo en el Sistema General de Riesgos Laborales. Bogotá: Ministerio de Trabajo; 2013. Disponible en: http:/ / ccs.org.co/salaprensa/images / Documentos/INFORME_EJECUTIVO_ II\%20ENCSST.pdf Acceso el 5 de noviembre de 2017

44. Martínez D, Crego A. I Encuesta sobre condiciones de trabajo, seguridad y salud laboral en Uruguay. Informe general. 2013. Disponible en: http://www.oiss.org/ estrategia/IMG/pdf/Encuesta_Uruguay. pdf Acceso el 8 de diciembre de 2017.

45. Instituto Brasileiro de Geografia e Estatística. Pesquisa Nacional de Saúde. Disponible en: https:/ /ww2.ibge.gov.br/ home/estatistica/populacao/pns/2013 vol4/default.shtm Acceso el 18 de julio de 2018.

46. Superintendencia de Riesgos del Trabajo. Micrositio. Estadísticas. Disponible en: https:/ / www.srt.gob.ar/index.php/ estadisticas-srt/ Acceso el 4 de junio de 2018

47. Secretaria de Previdência Social do Brasil. Ministério da Fazenda. Dados abertos - Saúde e segurança do trabalhador. Disponible en: http://www. previdencia.gov.br/dados-abertos/dadosabertos-sst/ Acceso el 4 de junio de 2018.

48. Superintendencia de Seguridad Social de Chile. Accidentes de Trabajo y Enfermedades Profesionales. Disponible en: http: / / www.suseso.cl/608/w3propertyvalue-59606.html Acceso el 4 de junio de 2018.

49. Fondo de Riesgos Laborales de la República de Colombia. Estadísticas 2017. Ministerio del Trabajo, 2015. Disponible en: http:/ / fondoriesgoslaborales.gov. $\mathrm{co} /$ seccion/informacion-estadistica/ estadisticas-2017.html\# Acceso el 4 de junio de 2018

50. Consejo de Salud Ocupacional. Publicaciones. Consejo de Salud Ocupacional, 2014. Disponible en: www.cso.go.cr/ documentos/publicaciones.html Acceso el 4 de junio de 2018.

51. Instituto Ecuatoriano de Seguridad Social. Estadísticas del Seguro de Riesgos del Trabajo. Disponible en: http://www.sart. iess.gob.ec/SRGP/indicadores_ecuador. php Acceso el 4 de junio de 2018. 
52. Instituto Salvadoreño de Seguridad Social. Estadísticas. Disponible en: http:/ / www.transparencia.gob.sv/institutions/ isss / documents / estadisticas? utf $8=\%$ E2\%9C\%93\&q[name_or_description cont]=actividad +\&q[year_cont] = 2017\&button $=\& q[$ document_category_ id_eq]=8697 Acceso el 4 de junio de 2017.

53. Instituto Hondureño de Seguridad Social (IHSS). Estadísticas del IHSS. Disponible en: http://ihss.hn/?page_id=3298 Acceso el 4 de junio de 2018.

54. Instituto Mexicano del Seguro Social (IMSS). Estadísticas e Informes. México: IMSS; 2017 Disponible en: www.imss.gob. $\mathrm{mx} /$ conoce-al-imss/informes-estadisticas Acceso el 4 de junio de 2018.

55. Instituto Nicaragüense de Seguridad Social. Estadísticas. Disponible en: https: / / www.inss.gob.ni/index.php / estadisticas-37 Acceso el 5 de junio de 2018.

56. Ministerio de Trabajo y Promoción del Empleo de Perú. Estadísticas Accidentes de Trabajo. Disponible en: www2.trabajo.gob.pe/estadisticas/estadisticasaccidentes-de-trabajo/ Acceso el 5 de junio de 2018.

57. Oficina Nacional de Estadística de República Dominicana. Accidentes laborales. Disponible en: www.one.gob.do/ sociales/sistema-dominicano-de-seguridad-social/accidentes-laborales Acceso el 5 de junio de 2018 .

58. Banco de Seguros del Estado de Uruguay. Informes mensuales. Disponible en: www. bse.com.uy/inicio/servicios/accidentesdel-trabajo/monitor-accidentes-del/informes-mensuales/ Acceso el 5 de junio de 2018.
59. Instituto Nacional de Prevención, Salud y Seguridad Laborales de la República Bolivariana de Venezuela. Estadísticas. Disponible en: http://www.inpsasel.gob. ve/moo_medios/sec_estadisticas.html\# Acceso el 5 de junio de 2018.

60. Ferrante D, Linetzky B, Konfino J, King A, Virgolini M, Laspiur S. Encuesta Nacional de Factores de Riesgo 2009: Evolución de la epidemia de enfermedades crónicas no transmisibles en Argentina. Estudio de corte transversal. Rev Argent Salud Publica. 2011;2(6):34-41.

61. Ferrante D, Virgolini M. Encuesta Nacional de Factores de Riesgo 2005: resultados principales: Prevalencia de factores de riesgo de enfermedades cardiovasculares en la Argentina. Rev Argent Cardiol. 2017;75(1):20-29. Disponible en: http:/ / www.scielo.org.ar/scielo.php? script=sci_arttext\&pid $=$ S1850-3748200 $7000100005 \& \operatorname{lng}=$ es Acceso el 25 de noviembre de 2017.

62. Ministerio de Salud de la Nación, Instituto Nacional de Estadísticas y Censos de la República Argentina. Tercera Encuesta Nacional de Factores de Riesgo para Enfermedades No Transmisibles; 2013. Disponible en: http://www.msal. gob.ar/images/stories/bes/graficos/ 0000000544cnt-2015_09_04_encuesta nacional_factores_riesgo.pdf Acceso el 20 de octubre de 2017.

63. Ministerio de Salud de Chile. Encuesta de Calidad de Vida y Salud (ENCAVI); 20152016. Ministerio de Salud, 2017. Disponible en: http://web.minsal.cl/wp-content/ uploads / 2017 / 02 / PRESENTACIONENCAVI-2016-11.02.2017.pdf Acceso el 15 de junio de 2017.
64. Ministerio de Salud de Chile. Encuesta Nacional de Salud (ENS). ENS 2016-2017. Ministerio de Salud, 2017. Disponible en: http:/ / epi.minsal.cl/informes-encuestaens/ Acceso el 15 de junio de 2017.

65. Organización Panamericana de la Salud/ Organización Mundial de la Salud (OPS/OMS). Enfermedades Transmisibles y Análisis de Salud/Información y Análisis de Salud. Base de Datos PLISA. Situación de Salud en las Américas: Indicadores Básicos 2017. Washington, D.C.: OPS/OMS; 2017. Disponible en: http://www.paho.org/ data/index.php/es/indicadores.html Acceso el 17 de octubre de 2017.

66. Eurofound. Sixth European Working Conditions Survey. Overview report. Luxembourg: Publications Office of the European Union; 2017. Disponible en: https: / / www.eurofound.europa.eu / sites/default/files/ef_publication/field ef_document/ef1634en.pdf Acceso el 17 de octubre de 2017.

67. Panorama Laboral 2013, América Latina y el Caribe. Lima: ILO/Regional Office for Latin America and the Caribbean; 2013. Disponible en: http://www.ilo.org/ wcmsp5/groups/public/---americas/--ro-lima / documents / publication / wcms_242634.pdf2305-0276 Acceso el 10 de setiembre de 2017.

Manuscrito recibido el 11 de diciembre de 2017. Aceptado para su publicación, tras revisión, el 11 de junio de 2018.
ABSTRACT

Proposed indicators for occupational health surveillance in Latin America and the Caribbean

This article proposes a set of common basic indicators for occupational health surveillance in the region of Latin America and the Caribbean and identifies the sources of data that are available for their measurement. To this end, consensus was built among members of the Expert Network on Working, Employment, and Health Conditions Surveys (RED ECoTES) in two stages. In the first stage, a preliminary list of indicators was agreed upon; in the second stage, data sources and their characteristics were identified to help determine the feasibility of measuring the actual value of each indicator in 20 countries of the region. The proposed set of basic indicators, together with their definitions, is composed of 13 indicators in four areas: employment conditions (three indicators), working conditions (four indicators), resources and preventive activities (two indicators), and health (four indicators). The primary sources of data available in the region for calculating these indicators were: permanent household surveys; surveys on conditions of work, employment, and health; vital statistics; and reporting systems for occupational injuries. Data are available in the region for many of the proposed indicators, but a sizable number of those having to do with working conditions and preventive activities are not available in most countries. For effective occupational health surveillance in the region, surveys on conditions of work, employment, and health must be vigorously promoted in each country.

Keywords

Health status indicators; epidemiological monitoring; information systems; occupational health. 
RESUMO

\section{Proposta de indicadores para vigilância em saúde ocupacional na América Latina e Caribe}

Palavras-chave
Neste artigo é proposto um conjunto básico de indicadores comuns para vigilância em saúde ocupacional na América Latina e Caribe, com a identificação das fontes de dados disponíveis para mensuração destes indicadores. A Rede Latino-Americana de Especialistas em Inquéritos sobre Condições de Trabalho, Emprego e Saúde (REDE ECoTES) conduziu um processo de decisão por consenso em duas etapas. A primeira etapa consistiu da seleção inicial dos indicadores e a segunda etapa compreendeu a identificação das fontes de dados e suas características, com o propósito de facilitar comprovar a viabilidade do cálculo efetivo de cada indicador em 20 países da Região. É proposto um conjunto básico de indicadores e respectivas definições contendo 13 indicadores distribuídos em quatro dimensões: condições de emprego (três indicadores), condições de trabalho (quatro indicadores) e recursos e atividades de prevenção (dois indicadores) e saúde (quatro indicadores). As principais fontes disponíveis na Região para determinar os indicadores são: as pesquisas permanentes de domicílios, as pesquisas sobre as condições de trabalho, emprego e saúde, as estatísticas vitais e os sistemas de notificação de acidentes de trabalho. Existem dados disponíveis para o cálculo de um número relevante dos indicadores propostos. Porém, a maioria dos países não dispõe de dados para calcular um número importante dos indicadores relacionados às condições de trabalho e atividades de prevenção. A vigilância efetiva em saúde ocupacional requer um firme incentivo às pesquisas sobre as condições de emprego, trabalho e saúde em cada país da Região.

Indicadores básicos de saúde; monitoramento epidemiológico; sistemas de informação; saúde do trabalhador. 